\title{
Pre-hospital transported patients - a resource for accessing prognostic risk factors
}

\author{
Camilla LN Bech ${ }^{1 *}$, Mikkel Brabrand ${ }^{1,2}$, Annmarie Lassen ${ }^{1}$ \\ From 6th Danish Emergency Medicine Conference \\ Odense, Denmark. 20-21 November 2014
}

\section{Background}

The survival of patients transported by ambulance depends not only on the clinical condition but also on other patient-related factors and the organizational prehospital setup. Until now, information on patients in the pre-hospital system has been almost unexplored. However, these data could form a valuable resource for assessing potential risk factors associated with adverse outcomes. Our aim was to describe ambulance transports to the Emergency Department and identify prognostic factors accessible in the pre-hospital phase and associated with seven-day mortality.

\section{Methods}

We included all adult patients ( $>18$ years) transported by ambulance to the Emergency Department at Odense University Hospital in the period $1^{\text {th }}$ of April 2012 to $30^{\text {th }}$ of September 2013. Ambulance personnel recorded vital signs and other clinical findings on a structured form on paper during the ambulance transport. Each contact was linked to information from population based healthcare registers in order to identify comorbid conditions and information on mortality. Demographic factors and first registered vital sign were analyzed by univariate logistic regression analysis with seven-day mortality as the outcome.

\section{Results}

In total, 24,620 ambulance contacts were identified. The median age of the patients was 61.4 years (IQR: 46-79), $49.5 \%$ were female, and $34.4 \%$ had severe comorbidity (defined as a Charlson Comorbidity Index $>=2$ ). Overall, seven-day mortality was 3.9\% (95\% CI: 3.6-4.1\%),

with 450 deceased females (3.9\%) and 499 deceased males (4.0\%). Univariate analyses revealed age, above 70 years; OR 16.2 (95\% CI: 8.61-30.3), Charlson score $\geq 2$; OR 2.65 (95\% CI 2.28-3.09), and vital parameters outside normal reference to be associated with sevenday mortality: Glasgow Coma Scale score $<14$ OR 8.62 (95\% CI: 7.52-9.89), peripheral oxygen saturation $<95 \%$ OR 2.75 (95\% CI 2.41-3.15), respiratory rate $>20 / \mathrm{min}$ OR 5.15 (95\% CI: 4.5-5.91), systolic blood pressure $<110 \mathrm{mmHg}$ OR 2.17 (95\% CI:2.17-3.35) and pulse $>90$ OR 2.47 (95\% CI: 2.16-2.83), respectively.

\section{Conclusions}

We found that several pre-hospital-registered vital signs recorded by ambulance personnel at first contact with the patient were prognostic factors of seven-day mortality.

\section{Authors' details}

'Department of Emergency Medicine, OUH Odense University Hospital, Odense, Denmark. Department of Emergency Medicine, Sydvestjysk Sygehus, Esbjerg, Denmark.

Published: 16 July 2015

\section{doi:10.1186/1757-7241-23-S1-A2}

Cite this article as: Bech et al: Pre-hospital transported patients - a resource for accessing prognostic risk factors. Scandinavian Journal of Trauma, Resuscitation and Emergency Medicine 2015 23(Suppl 1):A2.

\footnotetext{
* Correspondence: Camilla.noergaard.bech@rsyd.dk

'Department of Emergency Medicine, OUH Odense University Hospital,

Odense, Denmark

Full list of author information is available at the end of the article
} 